\title{
Suppression of airway inflammation by a natural acute infection of the intestinal epithelium
}

\author{
DL Gibbons $^{1,2}$, SFY Haque ${ }^{1}$, SL Copestake ${ }^{3}$, JW Wells $^{4}$, A Noble $^{4}$, AL Smith $^{3}$ and AC Hayday ${ }^{1,2}$
}

\begin{abstract}
Although chronic intestinal helminth infections may suppress allergen-induced airway pathology by inducing a combination of modified T-helper (Th) 2 and immunosuppressive cytokines, a similar capacity of natural acute intestinal infections has remained untested, despite their global prevalence. Here, we show that allergic airway phenotypes including eosinophilia, eotaxin mRNA, and Th2 cytokines are significantly suppressed in animals that were infected by and that have cleared the intestinal parasite Eimeria vermiformis. Unlike in helminth-infected animals, regulation requires temporal coincidence of infection with sensitization; depends on interferon- $\gamma$; and is not associated with an enhanced antigen-specific immunoglobulin G1 response. Moreover, regulation was effective following allergen sensitization in different anatomical sites, and in young and adult mice. These data highlight a transient anatomical dissemination of "functional immunologic dominance" following infection of the gut mucosa. They strongly support the hypothesis that airway allergies are naturally suppressed by both acute and chronic mucosal pathogens, but by different mechanisms.
\end{abstract}

\section{INTRODUCTION}

The increased prevalence of allergic diseases in the industrialized world over the past few decades almost certainly reflects an interaction of multiple genetic predispositions with changing environmental factors including diet, vitamin D exposure, and microbial milieu. With regard to the latter, Strachan ${ }^{1}$ proposed almost 20 years ago that the reduced exposure to childhood infections might decrease natural, antimicrobial T-helper (Th) 1 responses, with a corresponding immune deviation toward the Th2 phenotypes that characterize allergies such as airway hyperresponsiveness. Both epidemiological and experimental evidence seem to support this hypothesis. Thus, experimental models targeting the lung with Mycobacteria and Mycoplasma have shown suppression of allergy, ${ }^{2,3}$ seemingly consistent with a link of childhood bacillus calmette-guerin (BCG) vaccination to decreased allergy. ${ }^{4-6}$ Moreover, allergic pathology in different mouse asthma models can be both prevented and treated by immunostimulatory, CpG-containing, synthetic oliogodeoxynucleotides administered through different routes, albeit often at very high doses. ${ }^{7-10}$

Nonetheless, a substantially revised view has gradually developed based on epidemiologic, public health, and experimental studies of chronic worm infections that are widespread in the developing world. Experimental animal models have clearly illustrated that intestinal helminth infections may profoundly suppress allergic airway inflammation by eliciting both an "altered Th2 response", in which, for example, immunoglobulin G1 (IgG1) is more overt than IgE, and an immunosuppressive milieu, characterized by increased interleukin (IL)-10. ${ }^{11-15}$ These responses are consistent with the biology of helminths that stably exist in the host gut for long periods, yet sustain concomitant immunity toward new infections.

An attractive feature of the helminth studies is the capacity of the cytokine responses, induced by infection to also suppress Th-1-associated autoimmune diseases, that likewise are growing in frequency as the incidence of natural infections declines. Nonetheless, although a function for helminth infections in suppressing allergic and autoimmune pathologies seems uncontestable, additional issues deserve attention. First, not all helminth infections suppress allergy, with some strongly linked to exacerbation. Second, it is far from universally accepted that allergy suppression by helminths is representative of the way in which many other microbes may regulate allergies. ${ }^{16}$ In this regard, a third issue is the largely unresolved capacity (or otherwise) of acute resolving intestinal infections to regulate allergic airway inflammation. Like helminths, such infections are of extremely high prevalence. Thus, the World Health Organization states that Cryptosporidium is arguably the most frequently found intestinal pathogen in young children ( $1-5$ years) throughout the world,

\footnotetext{
${ }^{1}$ Programme in Infection and Immunity, Peter Gorer Department of Immunobiology, King's College London, Guy's Hospital, London, UK. Biomedical Research Centre, Guy's and St. Thomas' Hospital, King's College London, Guy's Hospital, London, UK. 'Enteric Immunology Group, Institute for Animal Health, Newbury, UK. ${ }^{4}$ MRC and Asthma UK Centre in Allergic Mechanisms of Asthma, King's College London, Guy's Hospital, London, UK. Correspondence: AC Hayday (adrian.hayday@kcl.ac.uk) 
and is equally common in infants of less than 1 year. Indeed, it is noteworthy that the World Health Organization quotes 95\% seroprevalence in South America, considering that Venezuela and Ecuador are two countries with conspicuously low levels of allergy. ${ }^{17}$ Thus, the prospect that acute intestinal infections may regulate allergies at distal anatomical sites, specifically the airways, merits closer examination. Furthermore, should such infections exert regulatory influence, does their mechanismof-action mimic that of helminth infections, or will it be exerted by a transient "functional immunologic dominance" over antigens encountered at approximately the same time?

To approach this issue, we have examined the influence on lung allergy of infection by Eimeria vermiformis, an apicomplexan parasite that naturally infects the murine intestine. Because of oral-fecal contamination in feeding, most young wild mice will be infected by Eimeria soon after weaning, a situation reflective of widespread natural parasitic infection by its close relative, Cryptosporidium parvum. Indeed, the prevalence of Eimeria infection in large mammals poses an ongoing burden to the agricultural industry.

E. vermiformis infects and replicates exclusively in enterocytes, and the disruption to the epithelial barrier manifests in lethargy, weight loss and related symptoms that rapidly become lethal as a single-dose escalates. However, because E. vermiformis cannot sustain more than a few rounds of mitotic division under reduced oxygen concentrations, it necessarily clears the gut between day 14 after infection (D14PI) and D20PI, even in immunodeficient hosts. ${ }^{18}$ In immunocompetent hosts, clearance is accelerated by interferon- $\gamma($ IFN $\gamma)$ dependent, Th1 responses mediated by T-cell receptor (TCR) $\alpha \beta+\mathrm{CD} 4+\mathrm{T}$ cells. Thus, parasite growth is significantly greater, and clearance greatly delayed in mice deficient for either IFN $\gamma$ or TCR $\alpha / \beta .{ }^{19,20}$ Wildtype (wt) mice acquire full immunity to rechallenge, remaining asymptomatic to reinfection by large parasite inocula that would be lethal in naïve animals. This immunity is completely absent in TCR $\alpha \beta$-deficient, knockout mice. ${ }^{20}$ However, primary immunoprotection in young mice shows a disproportionate dependence on $\gamma \delta$ T cells (reference ${ }^{21}$; DG and $\mathrm{AH}$, unpublished).

Gavage of laboratory mice can mimic natural Eimeria infection, which can then be accurately (and noninvasively) monitored by quantitating parasite oocysts in the fecal pellets. ${ }^{18,22}$ In this study, we have used this model of widespread natural acute gut infection to examine its influence on the airway response to an aerosolized antigen, ovalbumin (OVA), to which animals had been exposed, either intraperitoneally (IP) or epicutaneously, at the time of infection. As with chronic helminth infection, we find that acute Eimeria infection suppresses key components of the Th2-allergic response, notably IL-5 levels and eosinophilic inflammation. Nonetheless, the mechanisms-of-action and the host-parasite biology that evoke these common endpoints are clearly distinct in the two systems. Not only do the findings have important implications for human health, but they illustrate how the phenotype of the immune response to a natural acute intestinal infection transiently distorts the response to challenges at other mucosal sites. Operationally, we term this "functional immunologic dominance".

\section{RESULTS \\ Eimeria infection significantly reduces parameters of allergic airway inflammation}

Young C57BL/6 mice (28 days old) were infected at day 0 with E. vermiformis oocysts, following which allergic airway inflammation was induced in these and control mice employing a standard protocol using OVA as the allergen, as described in Figure 1a. Briefly, at D3PI, mice were sensitized by IP administration of OVA in alum. Sensitization was repeated 2 weeks later (D17PI). By D20PI at the latest, mice were completely cleared of the parasite, consistent with its acute need for reaeration for survival (references ${ }^{18,19}$ and data not shown). Note that alum sensitization did not affect the growth and clearance of the parasite relative to unmanipulated-infected mice (Figure 1b). Between 1 and 2 weeks after clearance, at D29PI, mice were challenged by a single-intranasal challenge of $100 \mu \mathrm{g}$ OVA or phosphate-buffered saline (PBS); and after a further $48 \mathrm{~h}$, immunologic parameters commonly associated with allergic asthma were assessed.

Figures 1 and 2 and Table 1 show the cumulative results obtained from several independent experiments. When compared to mock-sensitized mice, uninfected mice sensitized and challenged with OVA displayed significant infiltration into the bronchioalveolar lavage fluid (BALF) of leukocytes, including CCR3 ${ }^{+}$eosinophils (Figure 1c and d). Related to this, there was a conspicuously elevated level of eotaxin-1 and eotaxin-2 mRNA in the lungs (Figure 1e and data not shown). Eosinophilia is also known to be driven by IL- $5,{ }^{23}$ that was likewise significantly increased in the BALF (Figure 1f), along with other Th-2 cytokines, such as IL-4, IL-6, and IL-13 (Table 1). In aggregate, the changes observed mirror those reported in several other studies of allergic airway responses to aerosolized OVA. However, a very different result was obtained following OVA challenge of mice that had been OVA sensitized during oral infection by E. vermiformis that was subsequently cleared. Cellular infiltration into the BALF was suppressed, on average by twofold (Figure 1c; $P<0.022$ ), with greater than fourfold reduction in CCR $3^{+}$cells (Figure 1d; $P<0.02$ ). However, implying the selectivity of the effects of prior infection, the levels of macrophages and/or dendritic cells (DCs), as detected by major histocompatibility complex class II and CD11c expression, were not significantly different between the uninfected and the previously infected mice (data not shown). This contrasts sharply with the suppression of macrophage infiltration seen in OVA-challenged mice that are chronically infected with helminths.

Mice previously infected with Eimeria also showed suppression of eotaxin-2 and IL-5 mRNA expression (Figure 1e) and significant suppression $(P<0.008)$ of IL-5 protein (Figure 1f). It was also common for the levels of other Th2 cytokines (IL-4, IL-6, IL-13) to be strongly suppressed (Table 1) although on aggregate these did not reach statistical significance. In several individual experiments (Figure $\mathbf{1 g}$ and $\mathbf{h}$ ), this could be attributed to outlier mice (circled data points). Often (although not invariably), such outliers had also shed the lowest oocyst count of all infected animals in a group, probably reflecting a relatively weak infection. For example, the circled mouse in Figures If and $\mathbf{g}$ yielded 30 million oocysts compared to the group 
a

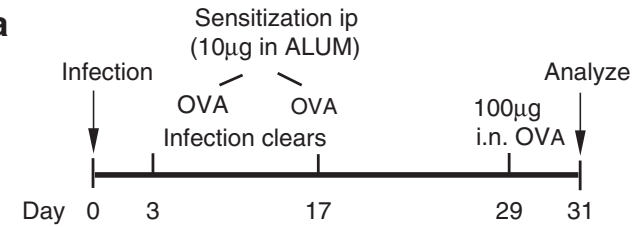

b

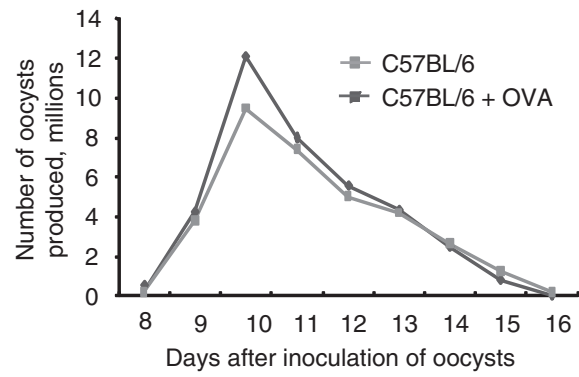

C
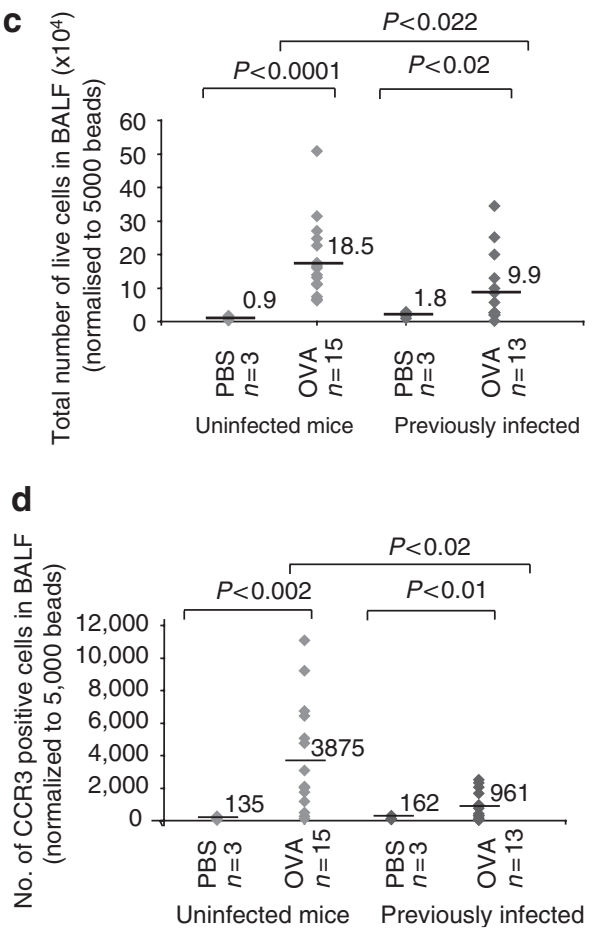

e

\begin{tabular}{l|l} 
Uninfected mice (OVA) & Previously infected (OVA)
\end{tabular}

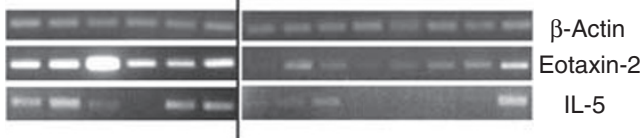

f
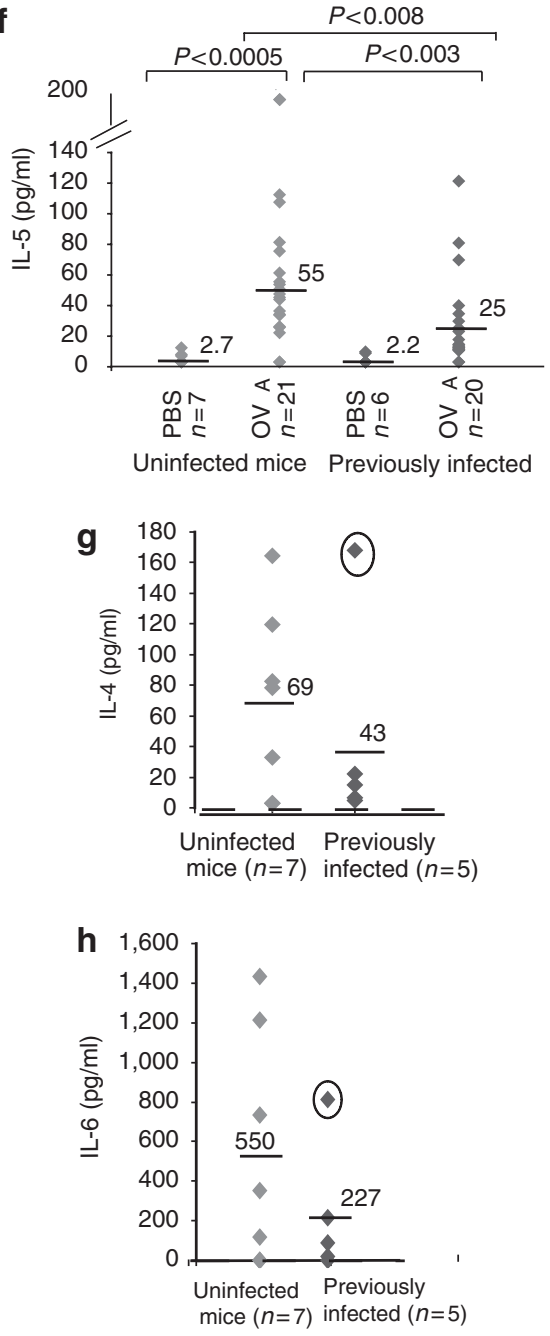

Figure 1 Prior infection with E. vermiformis inhibits airway reactions. (a) Main experimental protocol. Mice were infected at day 0 with oocysts by gavage. After 3 days, infected and control mice were sensitized intraperitoneally (IP) with $10 \mu \mathrm{g}$ OVA in ALUM and this was repeated at day 17. Mice were challenged on day 29 with $100 \mu \mathrm{g}$ OVA intranasally (i.n.) and culled 2 days later. Infected mice were housed separately and oocyst numbers determined from collected fecal pellets. (b) Parasite growth and clearance were comparable whether or not mice were simultaneously challenged with ova. Previously infected mice (diamonds, right hand side) or uninfected mice (diamonds, left hand side) subsequently sensitized and challenged with OVA or PBS from two independent experiments were assessed for total cell infiltration into the (c) BALF or (d) CCR3-positive cells (eosinophils) infiltrating into the BALF (normalized to 5,000 beads); levels of eotaxin and IL-5 mRNA in the lung from a representative experiment are shown in (e) with $\beta$-actin mRNA levels to indicate similar mRNA quantities; (f) protein levels in the BALF for IL-5 (cumulative data from three independent experiments, (g) IL-4 and (h) IL-6 (representative experiments) are also shown. The same outlier mouse (circled in $\mathbf{g}, \mathbf{h}$ ) showed the lowest oocyst count of the representative experiment shown.

average of $56 \mathrm{~m}$ (s.d. $14 \mathrm{~m})$. Notwithstanding some outliers, the clear downward trend in Th2 cytokine production (Table 1) again contrasted with the responses of allergen-challenged helminth-infected mice, where by contrast, the trend was for increased IL-4 production. ${ }^{12}$

Consistent with prior Eimeria infection promoting selective suppression of Th2 responses, most previously infected mice responded to OVA challenge with significantly higher levels $(P<0.032)$ of OVA-specific IgG2a, a T-dependent isotype whose production depends on ova-specific Th1-type activity (Figure 2a), and there was a significantly increased ratio of IgG2a: IgE compared to mice that had not been infected $(P<0.036)$. Again, this was not seen in challenged helminth-infected mice. Likewise, in mice previously infected with Eimeria there was 

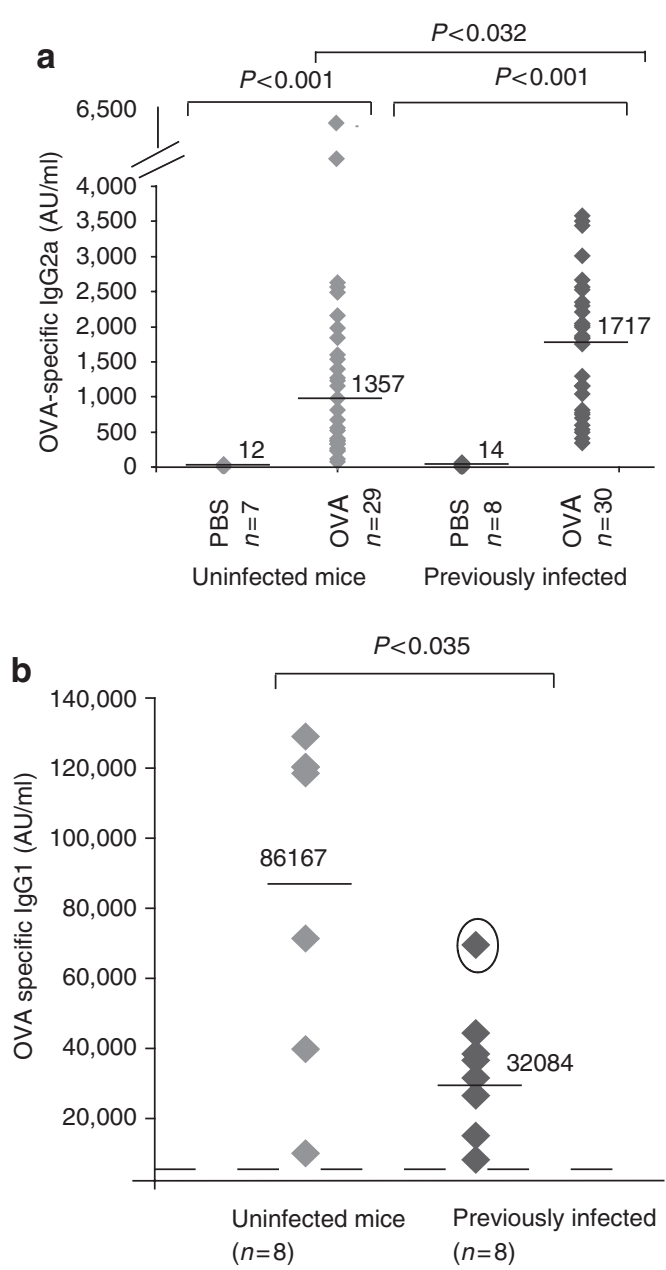

Figure 2 Prior infection with E. vermiformis alters OVA-specific Ig responses. Levels of OVA-specific IgG2a, detected by ELISA, are significantly increased in the serum of mice previously infected compared to those that were uninfected (a: cumulative data from four independent experiments). Levels of OVA-specific IgG1 are decreased in the serum of mice previously infected compared to those that were uninfected as shown in a representative experiment in (b). The dotted line represents average levels of OVA-specific IgG1 detected in PBS-treated mice. The outlier mouse (circled) showed the lowest oocyst count of the experiment shown. a clear downward trend in Th2-associated OVA-specific IgG1, for which an experimental group is shown in Figure 2b. Again, the circled mouse in which IgG1 suppression was the least, had yielded the lowest oocyst count, at $<50 \%$ of the mean. Such outliers contributed to IgG1 suppression not reaching statistical significance across all experiments, but the aggregate reduction of 1.6-fold compared to challenged uninfected mice, again contrasted sharply with OVA-challenged helminth-infected mice where there was a significant increase in OVA-specific IgG1, consistent with helminths promoting a "modified Th2 response". In addition, OVA-challenged helminth-infected mice showed significant increases in IL-10 relative to OVA-challenged uninfected mice, which was not the case for mice previously infected with Eimeria (data not shown). Conversely, neither example of infection-induced allergic suppression showed significant effects on IgE relative to OVA-challenged uninfected mice.

Collectively, the data demonstrate that gavage infection with Eimeria substantially affects the immune response to antigen sensitization performed at a distal site (IP). To test further the potential for anatomical spread of such "functional immunologic dominance", the study was repeated, but this time sensitizing mice to OVA in the skin by way of adjuvant-free epicutaneous patches ${ }^{24}$ (Figure 3a). As has been reported, subsequent intranasal challenge again induced various components of an allergic airway inflammatory phenotype, albeit that several parameters (particularly IL-6 induction) occurred to lesser degrees than in mice sensitized IP or in the lung. Nonetheless, despite the lower allergic response, key components of it, namely lung mRNA levels for both eotaxins were significantly suppressed $(P<0.032)$ in mice that were previously infected through the gut (Figures $\mathbf{3 b}$ and $\mathbf{c})$. There were likewise decreases of 1.5- to $>3$-fold in median levels of IL-4, IL-5, IL-6, and IL-13 (Figures 3d-f and data not shown). Again, the phenotype of outlier mice (circled) in each assay prevented the overall data set reaching statistical significance, but biologically it was noteworthy that the phenotypic outlier was again the animal yielding the lowest oocyst count before parasite clearance. The downward trend in all Th2 cytokines demonstrates that the general complexion of allergic suppression induced by acute gut infection is equivalent whether coincident sensitization occurs IP or epicutaneously, and is in

Table 1 Prior infection with E. vermiformis decreases Th2 cytokines in the BALF

\begin{tabular}{|c|c|c|c|c|c|}
\hline Cytokine (pg/ml) & \multicolumn{2}{|c|}{ Uninfected mice } & \multicolumn{2}{|c|}{ Previously infected mice } & $P$ value \\
\hline IL-4 & $5(2.1)$ & $33(10)$ & $1.6(0.7)$ & $21(8.8)$ & 0.2 \\
\hline IL-6 & $4.7(1.4)$ & $224(92)$ & $5.1(3)$ & $73(38.6)$ & 0.052 \\
\hline IL-13 (=s.e.m.) & $7.9(1.9)$ & $27(8.1)$ & $6.2(2.1)$ & $13(3.6)$ & 0.2 \\
\hline
\end{tabular}

IL, interleukin; OVA, ovalbumin; PBS, phosphate-buffered saline.

The mean level of cytokines (IL-4, IL-5, IL-6, and IL-13 all in pg/ml) measured in the BALF of OVA-challenged or PBS-challenged mice that were uninfected or previously infected with E. vermiformis is shown in this table. Data are pooled from three independent experiments. All cytokines were significantly induced in uninfected OVA-challenged vs. PBS-challenged mice and the levels of cytokines were all decreased following prior infection with E. vermiformis. The significance of the cytokine decrease following prior infection in OVA-treated mice is shown by the P value stated. s.e.m. values are shown in brackets. 
a
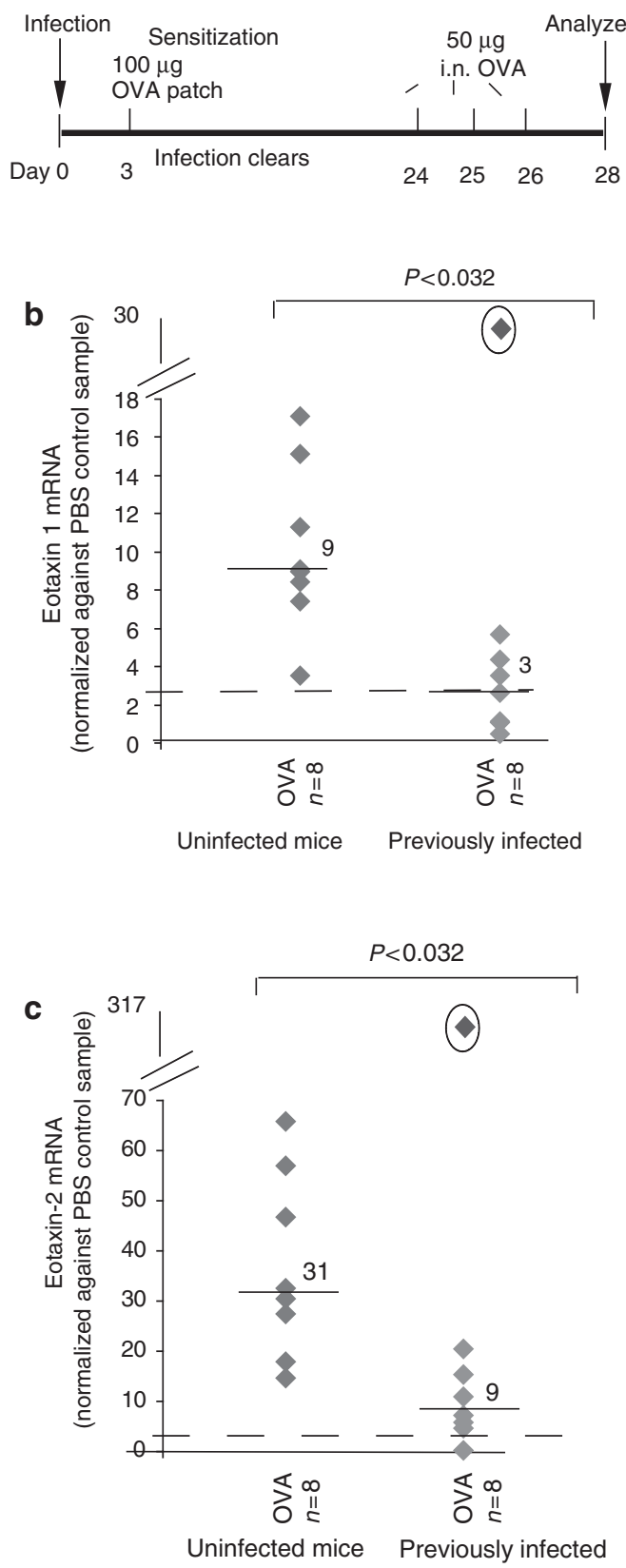
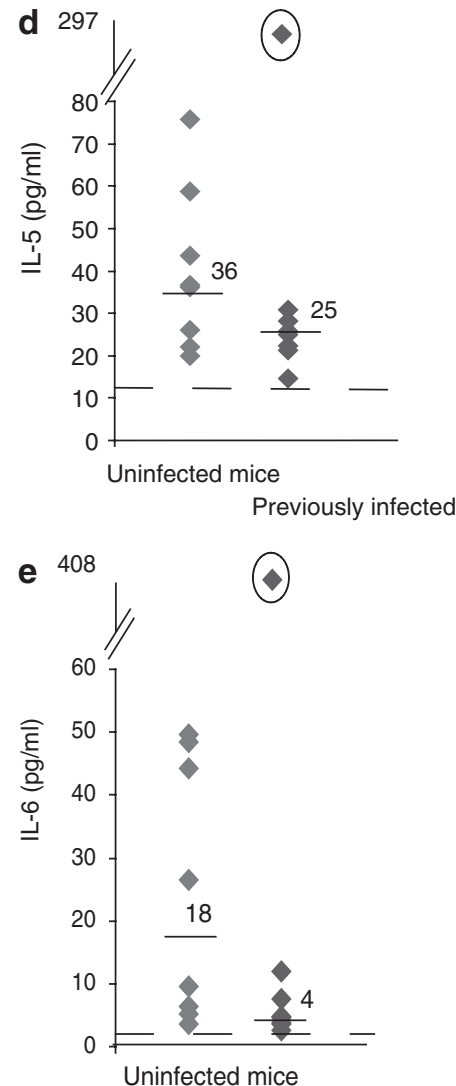

Previously infected

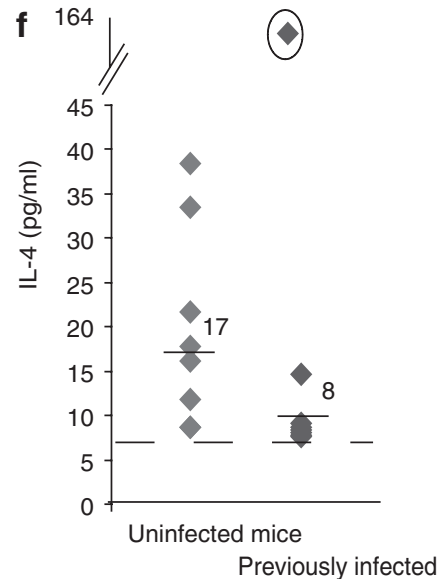

Figure 3 Prior infection with E. vermiformis inhibited allergy induced by epicutaneous sensitization. (a) The dosing regime. Eight mice were untreated or infected with 1,000 oocysts at day 0 and after shaving an occlusive patch was attached to the dorsal skin containing $100 \mu \mathrm{g}$ OVA or PBS at day 3 after infection. Mice were then challenged with $50 \mu \mathrm{g}$ OVA intranasally (i.n.) for 3 consecutive days before culling on day 28 . Levels of (b) eotaxin-1 and (c) eotaxin-2 mRNA, as determined by quantitative real-time PCR, are significantly decreased in mice previously infected (right, diamonds) compared to uninfected (left diamonds). Levels are normalized to a control mouse sample. (d-f) Levels of IL-5, IL-6 and IL-4 (respectively) detected in the BALF of uninfected (diamonds) or previously infected (diamonds) mice. The outlier mouse (circled) showed the lowest oocyst count of the experiment. In each graph, average expression levels for PBS-sensitized animals are shown as a dotted line and median levels of expression indicated.

both cases distinct from the form of allergy suppression associated with helminth infections.

\section{The protective effect of infection is not age dependent}

Consideration of the hygiene hypothesis frequently focuses on the exposure to infection of children. This is in part because allergic symptoms can be more profound in children and in young animals. Nonetheless, to determine whether the protective effects of $E$. vermiformis infection still operate in adult mice, infections with 100 E. vermiformis oocysts were performed either 1 week after weaning (28 days) or in adult mice (8-10 weeks old) that were then sensitized and challenged according to the 
regimen described in Figure 1a. For each parameter measured, the levels were essentially indistinguishable between mice infected and then challenged when either young or as adults, and these values were again significantly reduced vs. those in uninfected mice, as illustrated for eosinophil infiltration (Figure 4a), IL-5 expression (Figure 4b), and eotaxin-1 (Figure 4c). As these data reveal no obvious resistance to infection-induced allergy suppression in the adult, we hypothesize that the frequent association of the hygiene hypothesis with young individuals might represent a need for natural acute infection to occur coincident with initial environmental sensitization, an issue which we therefore tested.

\section{Protective effects of acute infection require temporal coincidence with sensitization}

To test if the timing of allergen sensitization relative to infection was important, an experiment was performed in which the initial OVA sensitization was delayed until D24PI (Figure 5a), about 1 week after clearance of E. vermiformis. In parallel, a cohort of animals was again sensitized at D3PI (as described above). Although there are natural quantitative variations in the parameters of allergic airway inflammation induced by each challenge experiment, delaying sensitization clearly had a dramatic effect on the outcome, as in no such case was there any significant reduction in any parameter of airway allergy that was measured (Figure 5b-d; Table 2). Likewise, there was no increase in OVA-specific IgG2a (Figure 5e) and no change in the OVA-specific IgG2a:IgE ratio. Thus, although airway immune effector responses can be substantively altered by a gut infection that has resolved before allergen challenge, this depends on the host having been sensitized to the antigen during infection.

\section{Protective effects of infection are IFN $\gamma$ dependent, but largely $\gamma \delta$ T-cell independent}

To investigate the mechanism underpinning allergy suppression induced by prior acute infection, similar experiments were performed in IFN $\gamma^{-/-}$mice. IFN $\gamma^{-1-}$ mice are significantly more susceptible to infection with E. vermiformis, ${ }^{19}$ and hence the parasite dose administered to IFN $\gamma^{-1-}$ and wt mice was adjusted (based on extensive experience) to achieve comparable levels of infection in both strains (10 oocysts in IFN $\gamma^{-/-}$ mice and 100 oocysts in wt mice (Figure 6a), which was a dose used previously to achieve significant allergic suppression (Figure 4). However, no such suppression was observed in infected IFN $\gamma^{-1-}$ mice (Figure 6). As a primary example, there were comparable levels of CCR3 ${ }^{+}$infiltrating cells in the BALF compared to uninfected mice (Figure 6b). Similarly, Th2 cytokine levels were not decreased by prior infection, and were actually slightly increased, consistent with immune activation in the context of there being no IFN $\gamma$ that ordinarily would antagonize Th2 responses (Table 3). Indeed, cytokine levels in the BALF of OVA-challenged IFN $\gamma^{-1-}$ mice were generally higher than those seen in wt C57BL/6 mice under the same conditions independent of infection, as illustrated by comparing Tables 1 and Table 3. Relative to wt mice, previously infected IFN $\gamma^{-/-}$mice also showed comparable levels of lung eotaxin-1
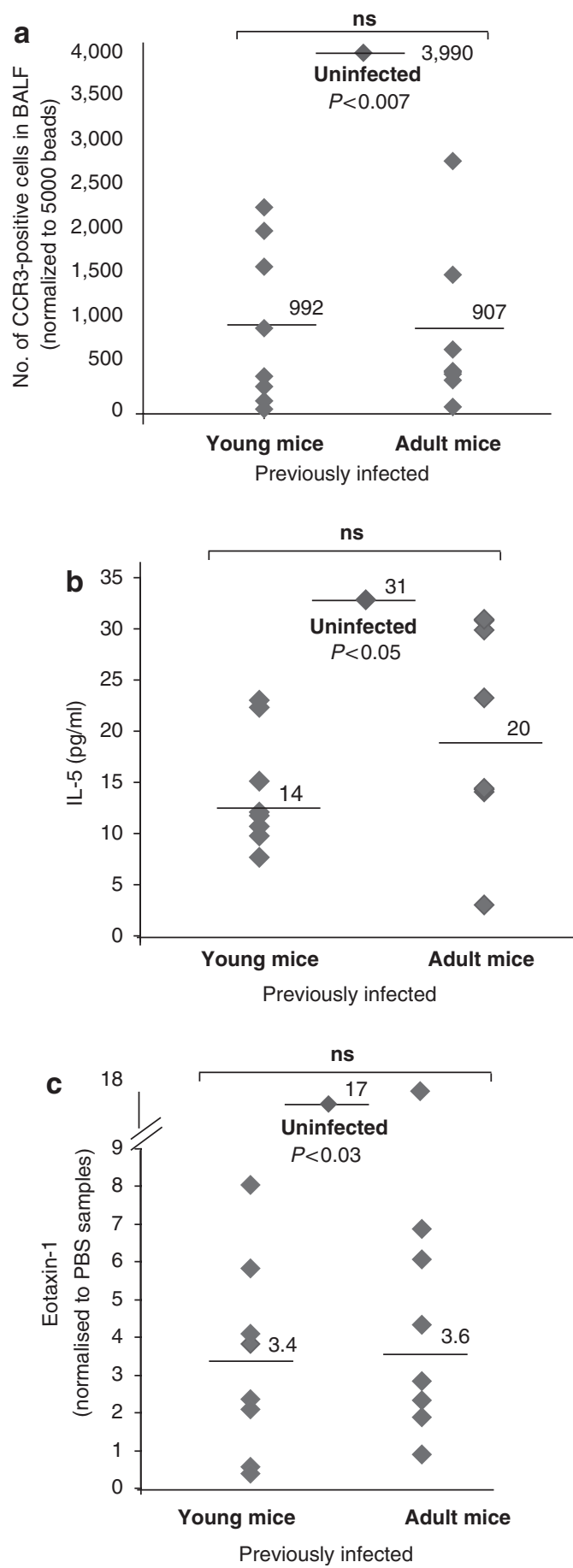

Figure 4 Infection of adult mice reduces allergic parameters. A total of 8 young mice ( 28 days old, left side) or 8 adult mice (8-10 weeks, right side) were infected with 100 oocysts at day 0 and then these mice and control mice were sensitized and challenged with OVA, as in Figure 1a. (a) Numbers of CCR3-positive cells infiltrating into the BALF, normalized to 5,000 beads, in infected young or adult mice as compared to the average levels of CCR3-positive cells seen in uninfected mice (diamond, middle). (b) Levels of IL-5 (pg/ml) in the BALF of infected young or adult mice in comparison to uninfected mice (diamond, center). (c) Levels of eotaxin-1 mRNA, as determined by quantitative PCR, in the lungs of young or old infected mice compared to uninfected mice (diamond, center). In each case, the $P$ value asserts a significant difference between uninfected mice and previously infected adult mice, which conversely show no significant difference vs. previously infected young mice. 
a

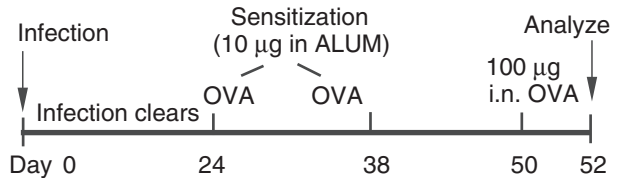

b
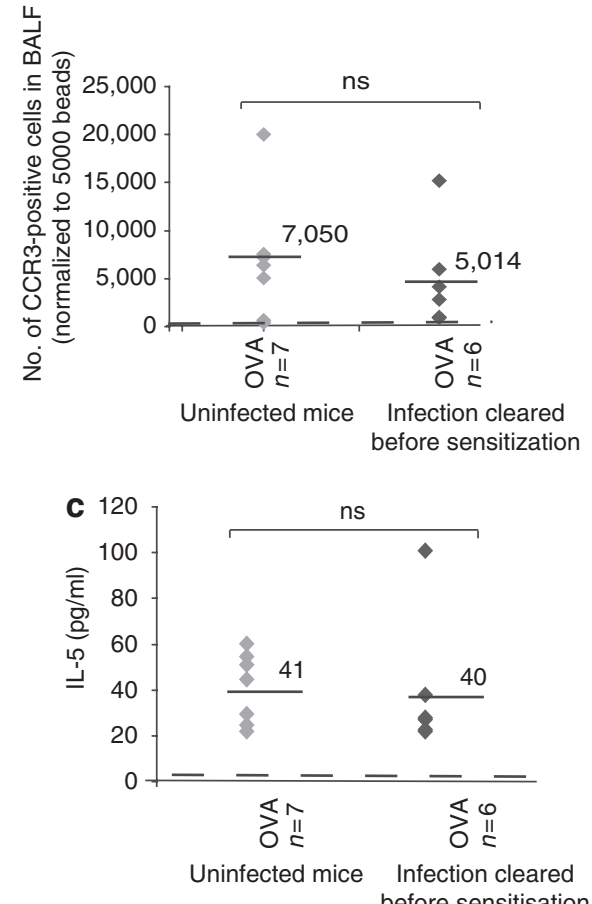

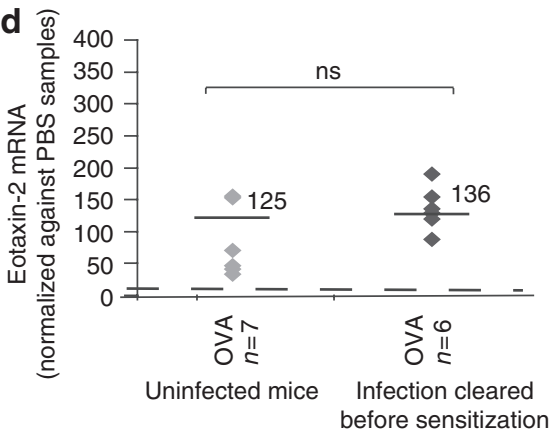

e
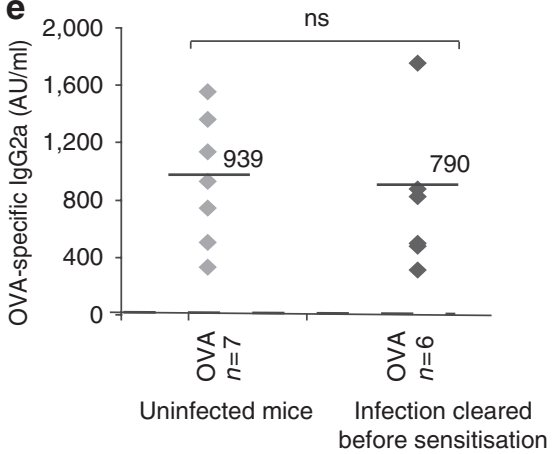

Figure 5 No protection is observed if mice are not sensitized while infected. (a) Experimental protocol for the delayed sensitization experiment where mice were infected at day 0 but then no sensitization was performed until day 24 and repeated at day 38 . Mice were challenged with $100 \mu g$ OVA on day 50 and culled at day 52. (b) Number of CCR3-positive cells (normalized to beads) infiltrating into the BALF of challenged mice previously infected with $E$. vermiformis but not sensitized until after the infection had cleared (6 mice, diamonds, right) compared to uninfected mice (7 mice, diamonds, left). (c) IL-5 levels in the BALF, (d) eotaxin-2 mRNA in the lung, or (e) OVA-specific IgG2a in the serum are shown from mice infected with E. vermiformis but then not sensitized until D24PI.

and -2 mRNA following OVA challenge (Figure $6 \mathbf{c}$ and data not shown). Collectively, these data demonstrate that the aggregate immune deviation induced by E. vermiformis infection completely depends on IFN $\gamma$ that is the major immunologic mediator of Th1-type host responsiveness to Eimeria infection. ${ }^{25}$

Considering that $\gamma \delta$ T cells might be the key source of such IFN $\gamma$ in young mice, the experimental protocol was repeated in $\mathrm{TCR} \delta^{-/-}$mice. Figure $\mathbf{6 d - f}$ shows that the suppressive effects of prior infection with $E$. vermiformis can be achieved independently of $\gamma \delta$ T cells. Levels of IL-5 (Figure 6d) and CCR3-positive cells (Figure 6e) in the BALF were clearly reduced in previously infected $\mathrm{TCR} \delta^{-1-}$ mice compared to uninfected $\mathrm{TCR} \delta^{-1-}$ mice and there was a significant reduction in eotaxin-1 mRNA levels in the lungs of previously infected TCR $\delta^{-1}$ mice compared to uninfected controls (Figure 6f). However, noting that the decreases in IL-5 and CCR3+ve infiltration did not reach significance it is possible that $\gamma \delta$ cells partially contribute to allergy suppression in this model.

\section{DISCUSSION}

From the diverse perspectives of basic immunology and human health, respectively, there has been much interest in the potential of chronic helminth infections to ameliorate allergies and Th1associated autoimmune diseases. The underlying mechanisms are not fully elucidated, but feature at least two components. ${ }^{14}$ First, there is the induction of a "modified Th2 response" characterized by high levels of IgG1 (mouse) or IgG4 (human). Such Th2 antibodies may functionally outweigh the effects of allergen-specific IgE, thereby reducing allergic pathology. The same mechanism may underlie the suppression of airway allergy by cat allergens. ${ }^{14}$ In addition, helminths induce a conspicuously immunosuppressive milieu that includes $\mathrm{CD} 25^{+}$T-reg cells, and that may involve IL-10. ${ }^{12,26,27}$ However, concern has been voiced about a mass acceptance of the view that helminth infections may be the sole paradigm for allergy suppression by natural gut infections. ${ }^{16}$ Indeed, the suppression of allergic airway inflammation by prior acute intestinal infection by Eimeria that we describe in this paper appears to be achieved in a different way. The infection-induced changes in cell counts, cytokines and Ig isotypes, and the dependence on IFN $\gamma$ all point to a mechanism more evocative of immune-deviation towards Th1 response, rather than a modified Th2 response. Moreover, although we have not directly tested the role of T-regulatory cells in Eimeriainduced allergy suppression, there is no logical reason to invoke 
Table 2 No protection is observed if mice are not sensitized although infected

\begin{tabular}{lcc}
\hline Cytokine (pg/mI) & $\begin{array}{c}\text { Infected mice } \\
\text { sensitized at day 3 } \\
\text { after infection ( } \boldsymbol{n = 5 )}\end{array}$ & $\begin{array}{c}\text { Infected mice } \\
\text { sensitized at day } \\
\mathbf{2 4} \text { after infection } \\
(\boldsymbol{n}=6)\end{array}$ \\
\hline IL-4 & $43(9.8)$ & $68(21.5)$ \\
IL-5 & $17(7.1)$ & $40(12)$ \\
IL-6 & $174(143)$ & $288(163)$ \\
IL-13 & $21(7.2)$ & $33(16.8)$ \\
IgG2a (Abs units) & $2,051(557)$ & $790(212)$ \\
(=s.e.m.) & &
\end{tabular}

IgG, immunoglobulin; IL, interleukin..

Mice were treated as in Figure 5a (delayed sensitization) or as in Figure 1a (sensitization during infection) and parameters of AAI assessed. In contrast to mice sensitized after infection had cleared (right panel), mice infected with E. vermiformis on the same day but sensitized at D3PI show much lower levels of these allergic parameters and increased levels of IgG2a.

them: for example, there is no known dependence of T-reg cells on IFN $\gamma$; there is no biological evidence for the induction of T-reg cells during acute intestinal infections by Eimeria; there is no change in IL-10 following OVA challenge of previously infected vs. uninfected mice; and the induction of long-lived T-reg cells does not fit the observation that allergy suppression by Eimeria infection requires that the schedules of sensitization and infection approximately coincide. In sum, allergy suppression by prior infection by Eimeria achieves the same endpoints-notably, significant suppression of IL-5 and eosinophilia-as does chronic worm infections, but by distinct mechanisms. This supports the view that there is not one single unifying mechanism by which pathogens regulate allergies and inflammatory diseases. Moreover, considering the very high global incidence of acute intestinal infections by pathogens such as Cryptosporidium, and considering their conservation it is reasonable to consider that the suppression of allergy by some form of Th2-Th1 immune deviation may be commonplace in the developing world, and may be conserved in many species.

Infection-induced Th2-Th1 immune deviation has been previously reported to suppress OVA-induced airway inflammation in the case of intranasal BCG administration. ${ }^{2}$ Likewise, intranasal inoculation with acinetobacter and lactococcus, respectively - two bacteria isolated from cowsheds-protected against airway eosinophilia in vivo, and in vitro upregulated the Notch ligand, Delta-like-4, on monocytes, which is associated with Th1 priming. ${ }^{28}$ Intranasal mycoplasma inoculation immediately before sensitization also upregulated Th1 cytokines and downregulated IL4 (although not to statistical significance). ${ }^{3}$ In each of these cases, regulation was local to the site of allergic pathology, and it was noticeable that where it was examined, there was no reduction in systemic Th2-type allergic parameters. ${ }^{2}$ By contrast, Eimeria infection demonstrates a capacity to regulate in trans from a site distal to both the site of priming and the site of allergic pathology.

It was previously reported that oral administration of immunostimulatory $\mathrm{CpG}$ oligodeoxynucleotides would suppress
OVA-induced allergic airway pathology, but the distinction between Th-1 immune deviation and T-reg-associated oral tolerance was not clearly made. ${ }^{8}$ Moreover, use of $\mathrm{CpG}$ is a reductionist approach that may usefully guide therapeutic approaches, but it does not address whether a natural infection by a complex gut pathogen will regulate airway allergy by promoting Th1 responses in trans. The mechanisms by which a gut infection can indeed exert "functional immunologic dominance" at different anatomical sites are unknown and clearly warrant elucidation, as they may reveal molecular and cellular conduits for rapid "immunologic information-sharing" between different anatomical sites. It is also not obvious a priori why one type of response in one site should transiently exert "functional immunologic dominance" over another. Perhaps in different situations, the "dominance hierarchy" would be reversed. Nonetheless, a similar situation was reported by Hussell and co-workers ${ }^{29}$ who noted that acute gut infection with Citrobacter rodentium can modulate the response to lung infection with Cryptococcus neoformans. Although that modulation was associated with Th1 skewing, no mechanistic analysis was provided.

The requirement for approximate coincidence of Eimeria infection and sensitization strongly suggests that priming is the direct target of infection-induced regulation, rather than the suppression of effector function that is achieved by chronic helminth infection. ${ }^{12,14}$ Thus the key to functional immunologic dominance across anatomical sites presumably resides in one or more cell types activated early in immune responses, possibly evoking a "burst of IFN $\gamma$ " in response to specific pathogens. Obvious candidates are DCs that may promote Th1 responses. By this scenario, the dependence of regulation on IFN $\gamma$ may reflect its promotion of IL-12 production by DC, perhaps by influence over the expression of Jagged and Delta-like ligands that reciprocally codetermine Th2/Th1 priming. ${ }^{30}$ IFN $\gamma$ also has a capacity to suppress the Th2 transcriptional program in $\mathrm{T}$ cells and to regulate Ig-isotype switching in B cells. Given that TLR stimulation of human DCs can lead to IFN $\gamma$ production by the DCs themselves, ${ }^{31}$ and given that DC derived IL-12, either alone or in combination with other cytokines, can also lead to induction of IFN $\gamma$ from murine DCs, ${ }^{32,33}$ it is also plausible that E. vermiformis stimulated DCs may themselves be a "transmissible" source of IFN $\gamma$.

However, there may be additional explanations. For example, it is possible that intestinal infections exert their effects through natural killer cells, which are a major source of IFN $\gamma$, and that can affect priming following migration to lymph nodes, as was described in a model system. ${ }^{34}$ Likewise, recent data have shown that subsets of CD8 T cells can release IFN $\gamma$ in response to cytokines alone, and that $\mathrm{CD} 8$ cells from mice previously infected with $E$. vermiformis produce elevated levels of IFN $\gamma^{35}$, raising them as a very strong candidate for involvement in immune deviation. In particular, they may be mobile. Conversely, we can exclude $\gamma \delta$ cells as the solo, nonredundant source of IFN $\gamma$, as the allergic airway phenotype induced in young $\mathrm{TCR} \delta^{-1-}$ mice by OVA sensitization and challenge was equivalent to that in wt mice (in contrast to some 

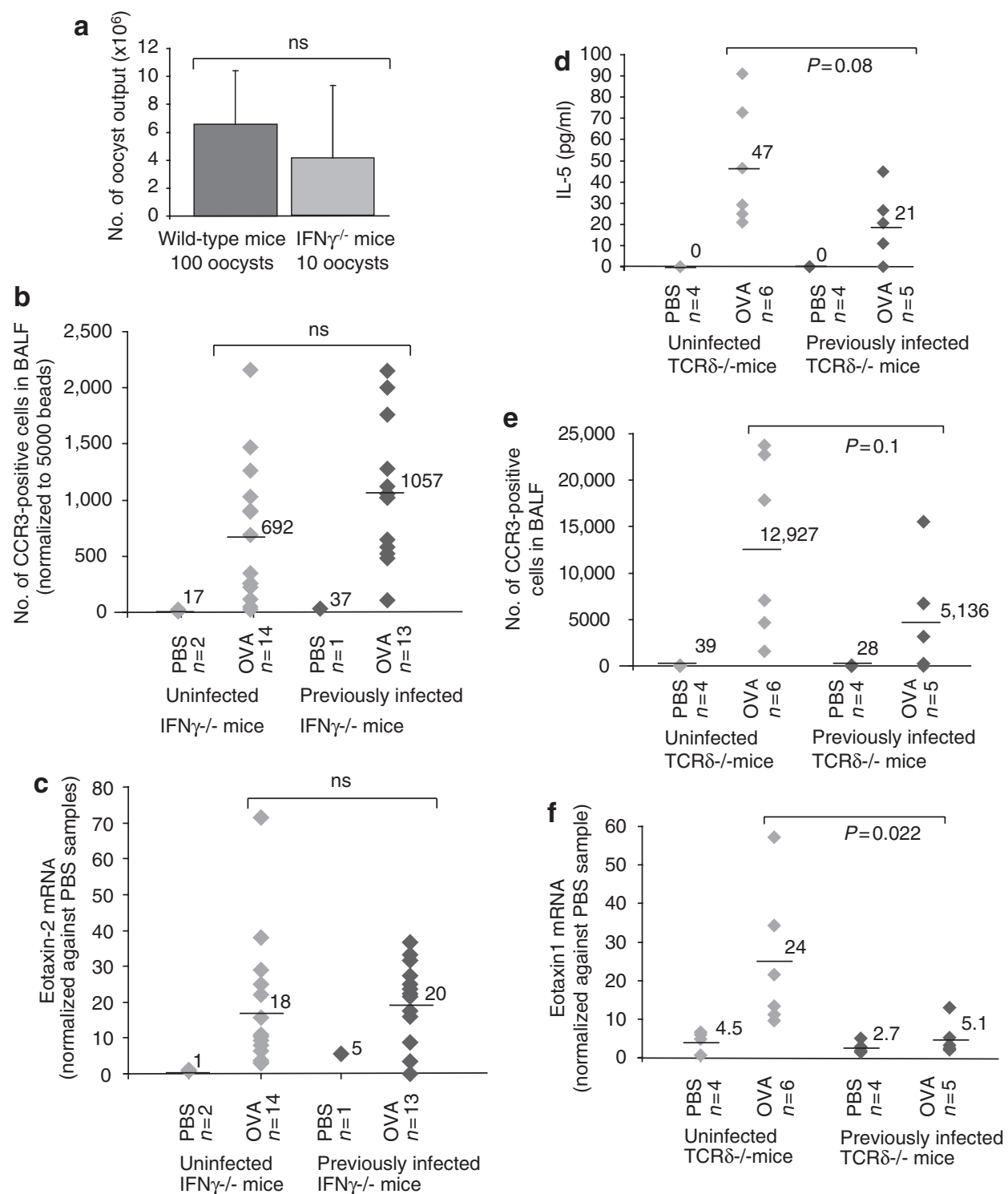

Figure 6 Allergy suppression by infection depends on IFN $\gamma$ but not on $\gamma \delta$ T cells. (a) Levels of oocysts collected from the feces of wt adult mice infected with 100 oocysts (left) or IFN $\gamma^{-l-}$ mice infected with 10 oocysts (right). A similar level of infection is seen in both. IFN $\gamma^{-1-}$ mice were, therefore, infected with 10 oocysts and OVA-sensitized and challenged as in Figure 1a. (b) Levels of CCR3-positive cells infiltrating into the BALF of OVA or PBStreated infected (diamonds, right) or uninfected (diamonds, left) IFN $\gamma^{-/-}$mice. Levels of eotaxin-2 mRNA in IFN $\gamma^{-/-}$mice infected (diamonds, right) or uninfected (diamonds, left) with E. vermiformis are shown in (c). Figure represents cumulative data from two independent experiments. TCR $\delta^{-/-}$ mice were infected, sensitized, and challenged as described in Figure 1a. Previously infected TCR $\delta^{-/-}$mice (diamonds, right hand side) or uninfected TCR $\delta^{-1-}$ mice (diamonds, left hand side) subsequently sensitized and challenged with OVA or PBS were assessed for (d) IL-5 levels in the BALF, (e) CCR3-positive cell infiltration into the BALF, and (f) eotaxin-1 mRNA in the lung. All AAI parameters tested were induced by OVA sensitization and challenge in the $\mathrm{TCR}^{-1-}$ mice and all were reduced by previous infection with $E$. vermiformis.

previous reports ${ }^{36,37}$ ), and was usually effectively reduced by infection with $E$. vermiformis (Figure 6d-f). Whatever cells are the initial source of IFN $\gamma$, the effect is clearly sustained as the antigen challenge to which there is a suppressed Th2 response and an increased IgG2a response, occurs weeks after infection.

One should also note IFN $\gamma$ may not be the only active agent in the form of immune deviation observed here. For example, Eimeria and other acute gut infections might have indirect effects, having provoked a significant change in the gut microenvironment. Data with respect to this point have yet to be obtained, but there are certainly many experiments demonstrating that changes in gut biota have wide-ranging effects on the immune system, both within and beyond the gut. ${ }^{38-41}$ This prospect only reinforces the view that gut-mediated, IFN $\gamma$ dependent mechanisms to regulate airway allergy will be vulnerable to changes in infection and diet that distinguish the developed from the developing world.

The finding that allergy regulation by Eimeria requires close temporal coincidence of infection and sensitization does not 
Table 3 Prior infection with E. vermiformis in IFN $\gamma^{-/-}$mice have no suppressive effect on Th2 cytokines in the BALF

\begin{tabular}{lcccc}
\hline \multirow{2}{*}{ Cytokine (pg/mI) } & \multicolumn{2}{c}{ Uninfected IFN $\gamma^{-/-}$mice } & \multicolumn{2}{c}{ Previously infected IFN $\gamma^{-/-}$mice } \\
\cline { 2 - 5 } & PBS (n=2) & OVA (n=14) & PBS (n=1) & OVA (n=13) \\
\hline IL-4 & $1.9(1.9)$ & $47(16)$ & 0 & $94(24.5)$ \\
IL-5 & $0(0)$ & $158(42)$ & 0 & $217(44)$ \\
IL-6 & $5.3(4.4)$ & $341(124)$ & 9.9 & $595(169)$ \\
IL-13 (=s.e.m.) & $0(0)$ & $204(72)$ & 0 & $274(68)$ \\
\hline
\end{tabular}

IFN $\gamma$, interferon- $\gamma$; IL, interleukin; OVA, ovalbumin; PBS, phosphate-buffered saline.

Mean levels of cytokines $(\mathrm{pg} / \mathrm{ml})$ measured in the BALF of OVA-challenged or PBS-challenged IFN $\gamma^{-/-}$mice (two independent experiments) that were uninfected or previously infected with E. vermiformis are shown in this table. All cytokines were significantly induced in OVA-challenged vs. PBS-challenged mice but there was no significant decrease in levels following prior infection with E. vermiformis; s.e.m. values are shown in brackets.

diminish its importance, as the alteration of the allergic response manifests upon allergen challenge that occurs after the infection has been cleared. Indeed, a need for infection to coincide with initial sensitization is in keeping with epidemiologic data that a developmental time window exists during which time exposure to the 'protective' microbial components must occur. ${ }^{42,43}$ Moreover, other data sets show that endotoxin or intranasal Mycoplasma pneumoniae infection could inhibit allergic pathology when exposure coincides with priming, but would actually exacerbate it if exposure post-dated sensitization. ${ }^{3,44}$ Future studies can address this issue in more detail by assessing over how long a time period the effects of prior acute Eimeria infection continue to suppress responses to subsequent allergic challenge. The importance of a "strong infection" in exerting regulation is suggested by the finding that animals that had yielded the lowest parasite counts during infection were often outliers, showing the lowest suppression of allergic parameters. Thus, this form of regulation might not be easily recapitulated with molecular mimics. Nonetheless, the temptation for clinical application is obvious, particularly given the capacity of acute gut infection to affect epicutaneous priming that is increasingly suspected to be a major means of sensitizing to airway allergens. The prospect that regulation could be achieved with an acute administration vs. chronic dosing is likewise attractive. Future experiments should determine the degree to which the pathogenicity of a natural gut pathogen may be attenuated while retaining its immunoregulatory potency.

\section{METHODS}

Mice. C57BL/6 (B6) mice (either 28 days' or 8-10 weeks' old) were purchased from Harlan, Oxford, UK. IFN $\gamma^{-1-}$ mice were purchased from Jackson Laboratory (Bar Harbour, ME) and bred locally. All protocols complied with UK Home Office regulations and were approved by Animal Welfare Committees on both sites.

Infection with E. vermiformis. E. vermiformis parasites were propagated and counted as described previously. ${ }^{18}$ Mice were infected by oral gavage of sporulated oocysts (between 10 and 1,000) in $0.2 \mathrm{ml}$ of water and fecal samples from individual mice collected daily from day 7 after infection, for enumeration of oocysts. Fecal samples were homogenized and diluted in saturated salt before microscopic evaluation of oocysts in a McMaster chamber. Daily counts were taken until no further oocysts were produced.
Induction of airway inflammation. Airway inflammation was achieved by either IP or epicutaneous sensitization with OVA followed by challenge. For IP sensitization, two injections of $10 \mu \mathrm{g}$ OVA (complexed with $1.8 \mathrm{mg}$ alum) were performed 14 days apart. Control mice received alum and PBS alone. Mice were subsequently challenged intranasally with either PBS (control mice) or $100 \mu \mathrm{g}$ OVA (in $40 \mu \mathrm{l}$ ) at day 26 after first immunization. At $48 \mathrm{~h}$ after the allergen exposure, mice were culled and assessed for parameters of allergic inflammation. For epicutaneous sensitization, mice were sensitized as described previously. ${ }^{24}$ Briefly, mice were shaved with electric clippers under anesthesia and then an occlusive patch (BandAid, Arlington, TX) containing either PBS or $100 \mu \mathrm{g}$ OVA was attached onto the shaved back. Patches remained in place for at least 3 days. Mice were challenged with $50 \mu \mathrm{g}$ OVA intranasally on 3 consecutive days beginning at day 25 PI. Mice were culled $48 \mathrm{~h}$ after the last challenge.

Preparation of ovalbumin. Mice were initially given purified OVA (Sigma; St Louis, MO, Grade V). In subsequent experiments, endotoxinfree OVA was prepared from fresh free-range chicken eggs (Lion Quality grade). Under sterile conditions, egg white was extracted using a $19 \mathrm{~g}$ needle and PBS added to give a $30 \mathrm{ml}$ solution. After vortexing (10 min), the volume was increased to $114 \mathrm{ml}(\sim 50 \mathrm{mg} / \mathrm{ml})$ and passed through a $0.2 \mu \mathrm{m}$ filter. Optical density was measured at $A_{280 \mathrm{~nm}}$, and the concentration of OVA calculated, assuming $54 \%$ of egg white is OVA. There was no endotoxin contamination. Preparations were stored at $-80^{\circ} \mathrm{C}$.

Analysis of bronchoalveolar lavage fluid. Animals were culled and BALF collected by flushing the lungs once with $1 \mathrm{ml}$ of PBS after cannulation of the trachea. The lavage fluid was centrifuged at $400 \mathrm{~g}$ for $5 \mathrm{~min}$ at $4{ }^{\circ} \mathrm{C}$ and the cells were separated from the fluid. The fluid was stored at $-20^{\circ} \mathrm{C}$ until analysis. The cells were immediately resuspended in fluorescence-activated cell sorting buffer containing PBS and $2 \%$ fetal calf serum (PAA). Inflammatory cells were analyzed by flow cytometry as described, ${ }^{45}$ with minor modifications. Briefly, cells were blocked with Fc $\gamma$ RIII/II block (BD Biosciences, San Jose, CA) for $15 \mathrm{~min}$ on ice. Cells were then stained with specific antibodies in the dark for $30 \mathrm{~min}$ on ice. Antibodies used were anti-CCR3-PE (R\&D Systems, Minneapolis, MN), anti-major histocompatibility complex class II-FITC, anti-CD3e-APC Cy7, anti-B220-PerCpCy5.5, and antiCD11c-APC (all purchased from BD Biosciences, San Jose, CA) with appropriate controls. The cells were washed three times and fixed in $1 \%$ paraformaldehyde. Nonfluorescent beads were added to each sample before measurement and an equal number of beads were read on a sevencolor Cytomation MoFlo machine. Data were analyzed using Summit software.

Cytokine analysis. BALF $(50 \mu \mathrm{l})$ was analyzed for various cytokines using Luminex technology using beadmates kits purchased from Millipore, Billerica, MA. Experiments were performed following the manufacturer's protocol for an overnight incubation except that each kit 
was diluted to allow the measurement of two plates. Protein standards were run in duplicate on every plate and all samples were measured in duplicate. Mean fluorescent intensity was detected using a Luminex 100 machine and the concentration was calculated from the standard curve for each cytokine using StarStation software. Data within the linear range were accepted and the few samples outside the linear range were diluted and retested.

RNA isolation and mRNA analysis. Lung tissue was collected in RNAlater (Ambion, Austin, TX) and stored at $4{ }^{\circ} \mathrm{C}$. Tissue samples were homogenized in TRIzol reagent (Invitrogen Life Technologies, Carlsbad, $\mathrm{CA}$ ) and RNA isolated according to the manufacturer's instructions. Genomic DNA contamination was removed with DNAse treatment (Promega, Madison, WI). Total RNA was used to synthesize complementary DNA using the Superscript First-Strand Synthesis System (Invitrogen Life Technologies). PCR amplification for varying cycle numbers was performed for $30 \mathrm{~s}$ at $94^{\circ} \mathrm{C} 45 \mathrm{~s}$ at $56^{\circ} \mathrm{C}$ (IL-5), $58^{\circ} \mathrm{C}$ ( $\beta$-actin) or $60^{\circ} \mathrm{C}$ (eotaxin-2), and $30 \mathrm{~s}$ at $72^{\circ} \mathrm{C}$ after an initial denaturation step of $5 \mathrm{~min}$ at $94^{\circ} \mathrm{C}$ The PCR products were run on a $1.5 \%$ agarose gel and visualized by ethidium bromide staining. PCR primers were: IL-5, $5^{\prime}$-AC TCTCAGCTGTGTCTGGG-3' ${ }^{\prime} 5^{\prime}$-GCCCACTCTGTACTCATCA C- $3^{\prime} ; \beta$-actin, $5^{\prime}$-CCCAGCCATGTACGTTGCTA-3', 5' -TCACCGGA GTCCATCACGAT-3'; eotaxin-2, 5' -GCTGCACGTCCTTTATTTCC$3^{\prime}, 5^{\prime}$-CCCCTTTAGAAGGCTGGT TT-3'. Real-time quantitative PCR for eotaxin-1, eotaxin-2, and cyclophilin was performed using SYBR Green Master Mix (Applied Biosystems, Carlsbad, CA) with forward and reverse primers at a final concentration of $300 \mathrm{nM}$ in a reaction volume of $20 \mu$ l. Gene-specific PCR products were continuously measured by the 7900 HT Sequence Detection System (ABI Prism, Carlsbad, CA) during 40 cycles at $60^{\circ} \mathrm{C}$. Primers for quantitative PCR were as follows:

eotaxin-1 5' -TTCTATTCCTGCTGCTCACG GTCA-3',

eotaxin-1R 5'-GTGTTGGGGATCTTCTTACT GGTC-3',

eotaxin- $2 \mathrm{~F}$ 5' -TTTGAACTCTGAGCTGTGC CTGAC-3', eotaxin-2R 5' -AGGGGATGGTCACAGAATC TATGG-3',

cyclophilin-F 5'-CAAATGCTGGACCAAACAC AA-3',

cyclophilin-F 5' -CCATCCAGCCATTCAGTCT TG-3'

Data were evaluated using the $2^{-} \Delta \Delta^{\mathrm{CT}}$ method ${ }^{46}$ using the mRNA level of cyclophilin within each sample for normalization. Average expression from duplicate samples was plotted in relation to average expression of cyclophilin and each point was presented relative to a sample from a single control animal that was set to 1 .

Determination of OVA-specific antibody levels. Pooled blood was collected after kidney excision and sera stored at $-20^{\circ} \mathrm{C}$ until assayed. Serum OVA-specific IgE was assayed by enzyme-linked immunosorbent assay, using anti-IgE coating antibody (LOME-3, $3 \mu \mathrm{g} / \mathrm{ml}$; AbD Serotec, Oxford, UK) and detection with $3 \mu \mathrm{g} / \mathrm{ml}$ OVA-biotin (conjugated with sulfo-NHS biotinylation kit; Perbio, Waltham, MA). For OVA-specific IgG2a and IgG1 assays, plates were coated with OVA $(50 \mu \mathrm{g} / \mathrm{ml})$, diluted sera were added and anti-mouse IgG2a or anti-mouse IgG1 alkaline phosphatase conjugates (Serotec) were used for detection. Results are expressed as arbitrary units calibrated against a standard curve constructed from pooled serum.

Statistical analysis. Unpaired Student's $t$-tests were used for parametric data. Mann-Whitney was used for nonparametric data. $P$ values of $P<0.05$ were considered significant.

\section{ACKNOWLEDGMENTS}

This work was funded by the NIAID R0161799 (DG, AH, and YH), Wellcome Trust (AH), Medical Research Council and Asthma UK (JWW, AN), and BBSRC, DEFRA and Jenner Institute (SC, AS). DG is currently supported by Cerimon Inc. We are grateful to Andy Archer for technical assistance and Wayne Turnbull for flow cytometry.

\section{DISCLOSURE}

The authors declare no conflicts of interest.

(C) 2009 Society for Mucosal Immunology

\section{REFERENCES}

1. Strachan, D.P. Hay fever, hygiene, and household size. BMJ 299, 1259-60 (1989).

2. Erb, K.J., Holloway, J.W., Sobeck, A., Moll, H. \& Le Gros, G. Infection of mice with Mycobacterium bovis-Bacillus Calmette-Guerin (BCG) suppresses allergen-induced airway eosinophilia. J. Exp. Med. 187, 561-9 (1998).

3. Chu, H.W., Honour, J.M., Rawlinson, C.A., Harbeck, R.J. \& Martin, R.J. Effects of respiratory Mycoplasma pneumoniae infection on allergeninduced bronchial hyperresponsiveness and lung inflammation in mice. Infect. Immun. 71, 1520-6 (2003).

4. da Cunha, S.S. et al. Lower prevalence of reported asthma in adolescents with symptoms of rhinitis that received neonatal BCG. Allergy 59, 857-62 (2004).

5. Linehan, M.F. et al. Is the prevalence of wheeze in children altered by neonatal BCG vaccination? J. Allergy Clin. Immunol. 119, 1079-85 (2007).

6. Marks, G.B. et al. The effect of neonatal BCG vaccination on atopy and asthma at age 7 to 14 years: an historical cohort study in a community with a very low prevalence of tuberculosis infection and a high prevalence of atopic disease. J. Allergy Clin. Immunol. 111, 541-9 (2003).

7. Kline, J.N., Kitagaki, K., Businga, T.R. \& Jain, V.V. Treatment of established asthma in a murine model using CpG oligodeoxynucleotides. Am. J. Physiol. Lung Cell Mol. Physiol. 283, L170-9 (2002).

8. Kitagaki, K., Businga, T.R. \& Kline, J.N. Oral administration of CpG-ODNs suppresses antigen-induced asthma in mice. Clin. Exp. Immunol. 143, 249-59 (2005)

9. Santeliz, J.V., Van Nest, G., Traquina, P., Larsen, E. \& Wills-Karp, M. Amb a 1-linked CpG oligodeoxynucleotides reverse established airway hyperresponsiveness in a murine model of asthma. J. Allergy Clin. Immunol. 109, 455-62 (2002).

10. Sur, S. et al. Long term prevention of allergic lung inflammation in a mouse model of asthma by CpG oligodeoxynucleotides. J. Immunol. 162, 6284-93 (1999).

11. Maizels, R.M. \& Yazdanbakhsh, M. Immune regulation by helminth parasites: cellular and molecular mechanisms. Nat. Rev. Immunol. 3, 733-44 (2003).

12. Wilson, M.S., Taylor, M.D., Balic, A., Finney, C.A., Lamb, J.R. \& Maizels, R.M. Suppression of allergic airway inflammation by helminth-induced regulatory T cells. J. Exp. Med. 202, 1199-1212 (2005).

13. Kitagaki, K. et al. Intestinal helminths protect in a murine model of asthma. J. Immunol. 177, 1628-35 (2006).

14. Fallon, P.G. \& Mangan, N.E. Suppression of TH2-type allergic reactions by helminth infection. Nat. Rev. Immunol. 7, 220-230 (2007).

15. Yang, J. et al. Schistosoma japonicum egg antigens stimulate CD4 CD25 $\mathrm{T}$ cells and modulate airway inflammation in a murine model of asthma. Immunology 120, 8-18 (2007).

16. Romagnani, S. Coming back to a missing immune deviation as the main explanatory mechanism for the hygiene hypothesis. J. Allergy Clin. Immunol. 119, 1511-3 (2007).

17. Medema, G. et al. WHO Guidelines for Drinking Water Quality: Cryptosporidium, www.who.int/entity/water_sanitation_health/ gdwqrevision/cryptodraft2.pdf (2006).

18. Rose, M.E., Owen, D.G. \& Hesketh, P. Susceptibility to coccidiosis: effect of strain of mouse on reproduction of Eimeria vermiformis. Parasitology 88 (Part 1), 45-54 (1984).

19. Smith, A.L. \& Hayday, A.C. Genetic analysis of the essential components of the immunoprotective response to infection with Eimeria vermiformis. Int. J. Parsitol. 28, 1061-1069 (1998).

20. Roberts, S. et al. T cell receptor alpha-beta + and gamma-delta+ deficient mice display abnormal but distinct phenotypes toward a natural, widespread infection of the intestinal epithelium. Proc. Natl. Acad. Sci. USA 93, 11774-11779 (1996). 
21. Ramsburg, E., Tigelaar, R., Craft, J. \& Hayday, A. Age-dependent requirement for $\gamma \delta$ T cells in the primary but not secondary protective immune response against an intestinal parasite.

J. Exp. Med. 198, 1403-1414 (2003).

22. Findly, R.C., Roberts, S.J. \& Hayday, A.C. Dynamic response of murine gut intraepithelial $T$ cells after infection by the coccidian parasite Eimeria. Eur J Immunol 23, 2557-64 (1993).

23. Collins, P.D., Marleau, S., Griffiths-Johnson, D.A., Jose, P.J. \& Williams, T.J. Cooperation between interleukin- 5 and the chemokine eotaxin to induce eosinophil accumulation in vivo. J. Exp. Med. 182, 1169-74 (1995).

24. Herrick, C.A., MacLeod, H., Glusac, E., Tigelaar, R.E. \& Bottomly, K. Th2 responses induced by epicutaneous or inhalational protein exposure are differentially dependent on IL-4. J. Clin. Invest. 105, 765-75 (2000).

25. Smith, A.L. \& Hayday, A.C. Genetic dissection of primary and secondary responses to a widespread natural pathogen of the gut, Eimeria vermiformis. Infect. Immun. 68, 6273-80 (2000).

26. Kitagaki, K., Businga, T.R., Racila, D., Elliott, D.E., Weinstock, J.V. \& Kline, J.N. Intestinal helminths protect in a murine model of asthma. J. Immunol. 177, 1628-1635 (2006).

27. Yazdanbakhsh, M., Kremsner, P.G. \& van Ree, R. Allergy, parasites, and the hygiene hypothesis. Science 296, 490-494 (2002).

28. Debarry, J. et al. Acinetobacter /woffii and Lactococcus lactis strains isolated from farm cowsheds possess strong allergy-protective properties. J. Allergy Clin. Immunol. 119, 1514-21 (2007).

29. Williams, A.E., Edwards, L. \& Hussell, T. Colonic bacterial infection abrogates eosinophilic pulmonary disease. J. Infect. Dis. 193, 223-30 (2006).

30. Amsen, D. et al. Instruction of distinct CD4T helper cell fates by different notch ligands on antigen-presenting cells. Cell 117, 515-26 (2004).

31. Fricke, I. et al. Mycobacteria induce IFN-gamma production in human dendritic cells via triggering of TLR2. J. Immunol. 176, 5173-82 (2006).

32. Fukao, T., Matsuda, S. \& Koyasu, S. Synergistic effects of IL-4 and IL-18 on IL-12-dependent IFN-gamma production by dendritic cells. J. Immunol. 164, 64-71 (2000).

33. Stober, D., Schirmbeck, R. \& Reimann, J. IL-12/IL-18-dependent IFNgamma release by murine dendritic cells. J. Immunol. 167, 957-65 (2001).
34. Martin-Fontecha, A. et al. Induced recruitment of NK cells to lymph nodes provides IFN-gamma for $\mathrm{T}(\mathrm{H}) 1$ priming. Nat. Immunol. 5, 1260-5 (2004).

35. Leggat, J.A. et al. Innate responsiveness of CD8 memory T-cell populations nonspecifically inhibits allergic sensitization. J Allergy Clin Immunol 122, 1014-1021 (2008).

36. Zuany-Amorim, C. et al. Requirement for gammadelta T cells in allergic airway inflammation. Science 280, 1265-7 (1998).

37. Lahn, M. et al. Negative regulation of airway responsiveness that is dependent on gammadelta T cells and independent of alphabeta T cells. Nat. Med. 10, 1150-1156 (1999).

38. Mazmanian, S.K., Liu, C.H., Tzianabos, A.O. \& Kasper, D.L. An immunomodulatory molecule of symbiotic bacteria directs maturation of the host immune system. Cell 122, 107-118 (2005).

39. Noverr, M.C. \& Huffnagle, G.B. Does the microbiota regulate immune responses outside the gut? Trends Microbiol. 12, 562-568 (2004).

40. Kalliomaki, M., Salminen, S., Poussa, T., Arvilommi, H. \& Isolauri, E. Probiotics and prevention of atopic disease: 4-year follow-up of a randomised placebo-controlled trial. Lancet 361, 1869-71 (2003).

41. Rosenfeldt, V. et al. Effect of probiotic Lactobacillus strains in children with atopic dermatitis. J. Allergy Clin. Immunol. 111, 389-95 (2003).

42. Riedler, J. et al. Exposure to farming in early life and development of asthma and allergy: a cross-sectional survey. Lancet 358, 1129-1133 (2001).

43. Radon, K., Ehrenstein, V., Praml, G. \& Nowak, D. Childhood visits to animal buildings and atopic diseases in adulthood: an age-dependent relationship. Am. J. Ind. Med. 46, 349-56 (2004).

44. Delayre-Orthez, C., de Blay, F., Frossard, N. \& Pons, F. Dose-dependent effects of endotoxins on allergen sensitization and challenge in the mouse. Clin. Exp. Allergy 34, 1789-95 (2004).

45. van Rijt, L.S. et al. A rapid flow cytometric method for determining the cellular composition of bronchoalveolar lavage fluid cells in mouse models of asthma. J. Immunol. Methods 288, 111-21 (2004).

46. Livak, K.J. \& Schmittgen, T.D. Analysis of relative gene expression data using real-time quantitative PCR and the 2(-Delta Delta C(T)) method. Methods 25, 402-8 (2001). 